\title{
An octamer of enolase from Streptococcus suis
}

\author{
Qiong $\mathrm{Lu}^{1,2}$, Hao $\mathrm{Lu}^{1}$, Jianxun $\mathrm{Qi}^{1}$, Guangwen $\mathrm{Lu}^{1 \bowtie}$, George F Gao ${ }^{1,2,3,4 \bowtie}$ \\ ${ }^{1}$ CAS Key Laboratory of Pathogenic Microbiology and Immunology, Institute of Microbiology, Chinese Academy of Sciences, \\ Beijing 100101, China \\ ${ }^{2}$ Graduate University, Chinese Academy of Sciences, Beijing 100049, China \\ ${ }^{3}$ Research Network of Immunity and Health (RNIH), Beijing Institutes of Life Science, Chinese Academy of Sciences, Beijing \\ 100101, China \\ ${ }^{4}$ Laboratory of Protein Engineering and Vaccines, Tianjin Institute of Industrial Biotechnology, Chinese Academy of Sciences, \\ Tianjin 300308, China \\ $\triangle$ Correspondence: gaof@im.ac.cn (G. Gao), luguangwen2001@126.com (G. Lu) \\ Received May 26, 2010 Accepted August 30, 2012
}

\begin{abstract}
Enolase is a conserved cytoplasmic metalloenzyme existing universally in both eukaryotic and prokaryotic cells. The enzyme can also locate on the cell surface and bind to plasminogen, via which contributing to the mucosal surface localization of the bacterial pathogens and assisting the invasion into the host cells. The functions of the eukaryotic enzymes on the cell surface expression (including $\mathbf{T}$ cells, $B$ cells, neutrophils, monocytoes, neuronal cells and epithelial cells) are not known. Streptococcus suis serotype 2 (S. suis 2, SS2) is an important zoonotic pathogen which has recently caused two large-scale outbreaks in southern China with severe streptococcal toxic shock syndrome (STSS) never seen before in human sufferers. We recently identified the $\mathbf{S S 2}$ enolase as an important protective antigen which could protect mice from fatal S.suis 2 infection. In this study, a 2.4-angstrom structure of the SS2 enolase is solved, revealing an octameric arrangement in the crystal. We further demonstrated that the enzyme exists exclusively as an octamer in solution via a sedimentation assay. These results indicate that the octamer is the biological unit of SS2 enolase at least in vitro and most likely in vivo as well. This is, to our knowledge, the first comprehensive characterization of the SS2 enolase octamer both structurally and biophysically, and the second octamer enolase structure in addition to that of Streptococcus pneumoniae. We also investigated the plasminogen binding property of
\end{abstract}

the SS2 enzyme.

KEYWORDS enolase, octamer, Streptococcus suis, structure, plasminogen binding

\section{INTRODUCTION}

Streptococcus suis serotype 2 (S. suis 2, SS2) is a Gram positive pathogen which can infect both animals and humans (Ma et al., 2009; Feng et al., 2010). The bacterium has been identified as the causative agent of many serious human diseases including meningitis, septicemia, endocarditis, arthritis and the streptococcal toxic shock syndrome (STSS), which is of severely high mortality (Tang et al., 2006). Two large-scale outbreaks of SS2 infection, which plagued several provinces of China in 1998 and 2005, have posed great concerns over its potential future severe outbreak (Tang et al., 2006). In an incessant endeavor to understand the pathogenesis of SS2, various virulence factors have been thus-far characterized, such as the capsule polysaccharide (Smith et al., 1999), several sortases (Vanier et al., 2008; Wang et al., 2009; Lu et al., 2011a), a suilysin (Xu et al., 2010) and a serum opacity factor(Baums et al., 2006). In addition, several two component systems (TCSs) (Li et al., 2008; Han et al., 2012) and a transmissible $89 \mathrm{~K}$ pathogenecity island (PI) (Chen et al., 2007; Li et al., 2011) have also been identified as involved in the pathogenesis of SS2. Besides these, Enolase is also considered to be a virulence factor (Esgleas et al., 2008; Feng et al., 2009).

Enolase exists universally among living organisms. Phy- 
logenetically, enolase belongs to a member of the enolase superfamily with three subfamilies represented by mandelate racemase (EC 5.1.2.2), muconate cycloisomerase (EC 5.5.1.1) and enolase itself (Babbitt et al., 1996; Gerlt et al., 2005). Functionally, enolase is an important enzyme involved in the glycolytic pathways, and is thereby considered to be evolutionarily conserved. Like many other metallo-enzymes, e.g. the widely concerned NDM-1 (Guo et al., 2011; Zheng et al., 2011), the activity of enolase also requires divalent metals, in the presence of which the enzyme could catalyze reversible conversion between 2-phosphoglycerate and phosphoenolpyruvate (Gerlt et al., 2005). There are also reports showing that enolase could interact with RNase E (Nurmohamed et al., 2010), and is a component of the multi-enzyme RNA degradosome (Chandran and Luisi, 2006). As a predominant cytoplasmic enzyme, enolase has also been identified with abundant amounts on the surface of various eukaryotic and many prokaryotic cells (Seweryn et al., 2007), despite the protein being devoid of classical surface protein motifs such as the signal peptide and the membrane-spanning domains. In eukaryotic cells, enolase has been found on the cell surface of T cells, B cells, neutrophils, monocytoes, neuronal cells and epithelial cells (Pancholi, 2001), where its functions are not fully understood. Various studies showed that the bacterial surface-localized enolase can bind to plasminogen, thereby contributing to the bacterial colonization of mucosal surfaces and then invasion of the host cells (Jones and Holt, 2007; Feng et al., 2009). Therefore, enolase is also an important virulence factor in many bacterial pathogens.

Thus far, a series of enolase structures have been reported (Stec and Lebioda, 1990; Kuhnel and Luisi, 2001; Hosaka et al., 2003; Ehinger et al., 2004; Kang et al., 2008). All these structures reveal a similar protein fold with a small $\mathrm{N}$-terminal domain and a large C-terminal domain of TIM-barrel like. With a conserved overall structure for the enolase protein, the oligomeric state of the enzyme could be quite different in either a dimeric or an octameric forms (Stec and Lebioda, 1990; Kuhnel and Luisi, 2001; Ehinger et al., 2004; Kang et al., 2008). It has been proposed that most enolases exist as homodimers including those from all eukaryotes and many prokaryotes (Brown et al., 1998). In contrast, far less enolases have been demonstrated as octamers (Schurig et al., 1995; Ehinger et al., 2004; Karbassi et al., 2010), all of which are exclusively from the prokaryotic species. Therefore, the elucidation of the oligomeric state of an enolase is crucial for the characterization of the enzyme.

In our previous study, we already systematically investigated the functions of SS2 enolase (Feng et al., 2009). We found that apart from its conserved function in carbohydrate metabolism, the enzyme could also elicit strong humoral antibody responses and protect mice from fatal SS2 infection (Feng et al., 2009), thereby making the protein an important protective antigen. Nevertheless, the structural features and oligomeric state of this SS2 enzyme have not been fully characterized. In this study, we solved the crystal structure of SS2 enolase at a resolution of $2.4 \AA$. The overall structure of the SS2 enzyme resembles other reported enolase structures. Simple symmetry operations could yield an octameric structure as observed in that of the Streptococcus pneumoniae enolase. We further demonstrated that SS2 enolase indeed exists exclusively as an octamer in solution via ultracentrifugation sedimentation assay. This is, to our knowledge, the second octameric enolase structure after the $S$. pneumoniae enolase, yet the first comprehensive characterization of an enolase octamer both structurally and biophysically. These results provide solid evidence that an octamer is the biological unit of SS2 enolase at least in vitro and quite possibly in vivo as well. We also characterized the plasminogen binding property of the SS2 enzyme via surface plasmon resonance.

\section{RESULTS}

\section{The overall structure of SS2 enolase}

The functional importance of SS2 enolase in both the glycoIytic pathway and the bacterial virulence makes it of great interest to explore the structural features of this evolutionarily conserved enzyme (Feng et al., 2009). Our previous protein preparation of SS2 enolase with an N-terminal his-tag failed to yield any crystals in the initial screening trials. Therefore an alternative, with the purification tag being moved to the C-terminus, was constructed for crystallization, in which crystals of good quality were obtained. The structure was quickly determined by the molecular replacement method using the homologous structure of S.pneumoniae enolase (Ehinger et al., 2004) as an initial search model, since the two proteins share an overall identity of about $88 \%$ in the primary sequence (Fig. 1). The final model, with a resolution of $2.4 \AA$, has an $R_{\text {work }}$ factor of 0.139 and an $R_{\text {free }}$ factor of 0.162 , respectively. Overall, $95.0 \%$ of the residues located in the most favored region of the Ramachandran plot with $5.0 \%$ in the additionally and generously allowed region and no residues in the disallowed region, indicating a good stereochemistry for the structure (Table 1).

Within the crystallographic asymmetric unit of the structure, two enolase monomers are present which are tightly related via a two-fold axis. For each monomer, clear electron density could be observed for 428 amino acids extending from Met1 to Leu433, with terminal residues $434-435$ as well as loop residues $41-43$ and $252-253$ being invisible due to disorder. The two molecules of the asymmetric unit are basically the same structures with an R.M.S.D. of only about $0.2 \AA$ for all the $C \alpha$ pairs. Each molecule is sterically arranged into two domains (Fig. 2A). The small N-terminal domain is composed of residues 1-143, folding into a three-stranded anti-parallel $\beta$-sheet (S1, S2 and S3) and four intertwined a-helices $(H 1$, $\mathrm{H} 2, \mathrm{H} 3$ and $\mathrm{H} 4$ ). In contrast, the $\mathrm{C}$-terminal domain is much 

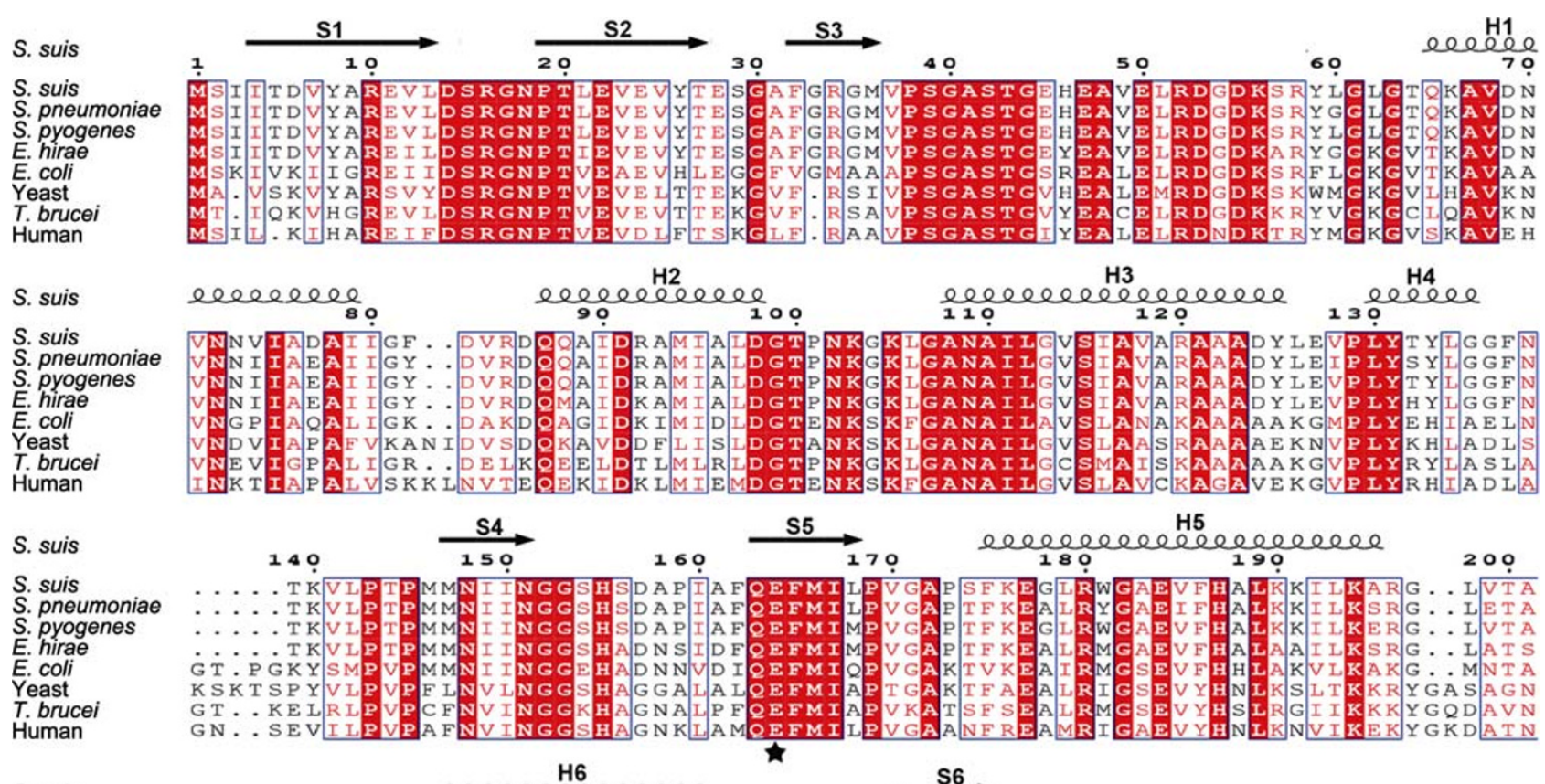

S. suis

S. suis

S. pneumoniae

S. pyogenes

E. hirae

Yeast

T. brucei

Human

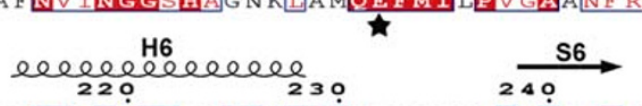

$210220 \quad 230$

250

260

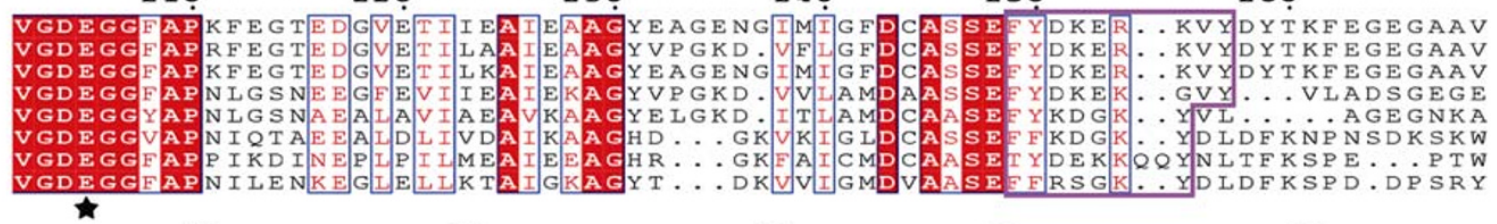

sase

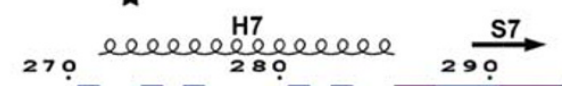

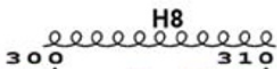

$\stackrel{S 8}{\longrightarrow}$ (20

eceelece

S. suis

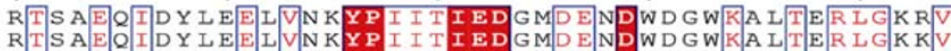

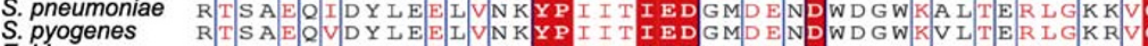

E. hirae

Y. coli

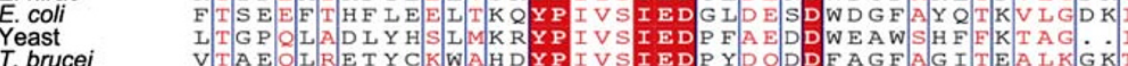

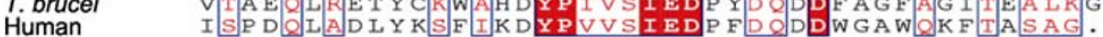

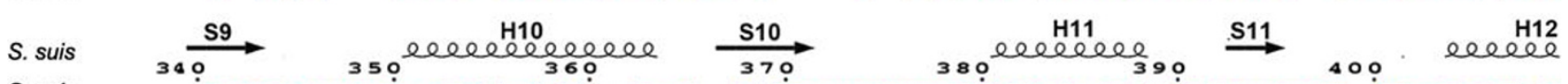

S. suis

S. pneumoniae

S. pyogenes

E. hirae

E. coli

T. brucei

Human

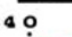

St

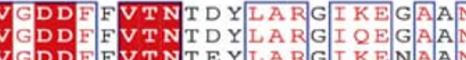
GDD I F V T N T Q K I S E G I I E K G I A $\checkmark G D D I$ F V T N N T K I I L K E G G I E K G

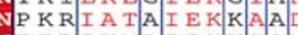
年

S. suis 410 oberele

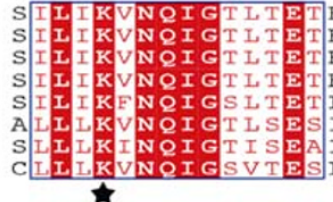

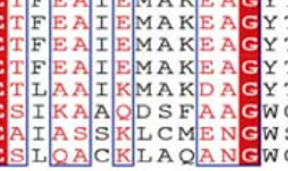
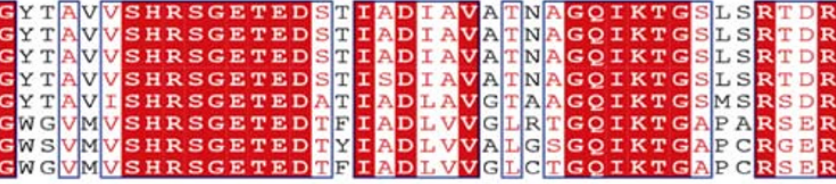

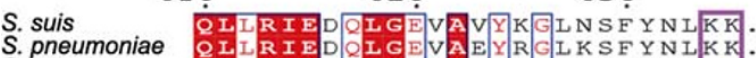

S. pyogenes QLIRIEDQLGEVAOY K GIK I S F Y NLKK.

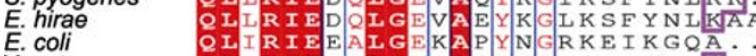

Yeast

Human

QLIRIEEEIGDNAVEAGENFHHGDAI:

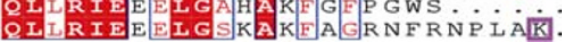

Figure 1. Multiple sequence alignment of enolases from representative prokaryotic and eukaryotic species. The enolases from Streptococcus suis, Streptococcus pneumonia, Streptococcus pyogenes, Enterococcus hirae, E.coli, yeast, Trypanosoma brucei and human are selected for comparison. The secondary structural elements are marked with spiral lines and horizontal arrows to represent $\alpha$-helices and $\beta$-strands, respectively, and labeled according to their occurrence in the structure. The active site residues of the catalytic centre are indicated by labeling with black stars, and the putative plasminogen binding sites are highlighted by enclosure in purple boxes. 
Table 1 Summary of crystal parameters, data collection and refinement statistics

\begin{tabular}{|c|c|}
\hline \multicolumn{2}{|l|}{ Data collection } \\
\hline Space group & 14 \\
\hline Unit cell parameters $(\AA)$ & $a=b=168.0, c=77.1$ \\
\hline Wavelength $(\AA)$ & 1.5418 \\
\hline Resolution range $(\AA)$ & $50-2.4(2.49-2.40)$ \\
\hline Number of total reflections & 297558 \\
\hline Number of unique reflections & 41166 \\
\hline Completeness (\%) & $98.3(96.8)$ \\
\hline$R_{\text {merge }}{ }^{a}$ & $0.062(0.312)$ \\
\hline Redundancy & $7.2(6.8)$ \\
\hline Mean $/ / \sigma$ & $46.9(7.2)$ \\
\hline \multicolumn{2}{|l|}{ Refinement statistic } \\
\hline$R_{\text {cryst }}{ }^{\mathrm{b}} / R_{\text {free }}{ }^{\mathrm{c}}$ & $0.141 / 0.162$ \\
\hline RMSD bonds $(\AA)$ & 0.002 \\
\hline RMSD angles $\left({ }^{\circ}\right)$ & 0.520 \\
\hline Number of protein atoms & 6517 \\
\hline Number of water molecules & 234 \\
\hline Mean $B$ values $\left(\AA^{2}\right)$ & 39.6 \\
\hline Ramachandran favored (\%) & 95.0 \\
\hline Ramachandran allowed (\%) & 5.0 \\
\hline
\end{tabular}

The values in parentheses are those for the highest resolution shell.

${ }^{a} R_{\text {merge }}=\Sigma_{h} \Sigma_{i} l_{i}(h)-<l(h)>\mid / \Sigma_{h} \Sigma_{i} l_{i}(h)$, where $l_{i}(h)$ is the intensity of an individual measurement of the reflection and $\langle l(h)\rangle$ is the mean intensity of the reflection.

${ }^{\mathrm{b}} R_{\text {cryst }}=\Sigma_{h}\left|F_{\text {obs }}-F_{\text {calc }}\right| / \Sigma_{h}\left|F_{\text {obs }}\right|$, where $F_{\text {obs }}$ and $F_{\text {calc }}$ are the observed and calculated structure factor amplitudes, respectively

${ }^{\mathrm{c}} R_{\text {free }}$ was calculated as $R_{\text {cryst }}$ using $5.0 \%$ of the randomly selected unique reflections that were omitted from structure refine

larger, with amino acids 144-433 forming an eight-stranded mixed $\alpha / \beta$-barrel. Strands S4-S11 are arranged in a parallel manner to constitute the core of the barrel, and eight large helices (H5-H12) sequentially surround the core strands to form the peripheral barrel wall (Figure 2A). This domain is also commonly designated as the barrel domain in other enolase structures (Stec and Lebioda, 1990; Kuhnel and Luisi, 2001; Hosaka et al., 2003; Ehinger et al., 2004), and is an atypical TIM-barrel fold (Wierenga, 2001) in terms of its topology and configuration. $A \beta_{2} \alpha_{2}(\beta \alpha)_{6}$ topology for the enolase $\mathrm{C}$-terminal domain differentiates it from the canonical TIM-barrel which exhibits a more regular $(\beta \alpha)_{8}$ composition as represented by triosephosphate isomerase (Banner et al., 1975). The putative active site of the catalytic centre in SS2 enoloase consists of residues Glu164, Glu205 and Lys344, corresponding to the catalytic amino acids Glu164, Glu205 and Lys342 in the S. pneumoniae counterpart. These three residues are located in the $\mathrm{S} 5 \beta$-strand, the $\mathrm{H} 5 / \mathrm{H} 6$ and $\mathrm{S} 9 / \mathrm{H} 10$ intervening loops, respectively, and are strictly conserved among all enolases (Fig. 1).

The SS2 enolase is quite similar in the 3-dimentinal structure to other reported enolases of either eukaryotic or prokaryotic species. A Dali search (Holm and Sander, 1996) within the protein data bank reveals a series of homologous structures, representatives of which including those from human, yeast, E.coli and $S$. peneumoniae are selected for superimposition (Stec and Lebioda, 1990; Kuhnel and Luisi, 2001; Ehinger et al., 2004; Kang et al., 2008). As shown in Fig. 2B, all the selected structures could be well aligned. The $S$. pneumoniae enolase exhibits the highest structural similarity with our enzyme with an R.M.S.D. of about $0.3 \AA$ for the matching $\mathrm{Ca}$ positions, while the other three yield relatively higher deviation values ranging from $0.6-1.0 \AA$. With a highly conserved main body structure, conformational difference is observed in two loop regions (Fig. 2B). One is the long linker connecting strand $\mathrm{S} 3$ and helix $\mathrm{H} 1$, another one is the intervening loop between strand $\mathrm{S} 6$ and helix $\mathrm{H} 7$, both of which are quite flexible in our structure (see results above). It is also noteworthy that the $\mathrm{S} 6 / \mathrm{H} 7$ loop has been indicated in the plasminogen binding of the $S$. pneumoniae enolase (Ehinger et al., 2004).

\section{Solid evidence of an enolase octamer both in crystal and in solution}

Enolase has been found to exist as a homodimer in all eukaryotes and many prokaryotes (Brown et al., 1998), although octameric enolases from several bacterial species 


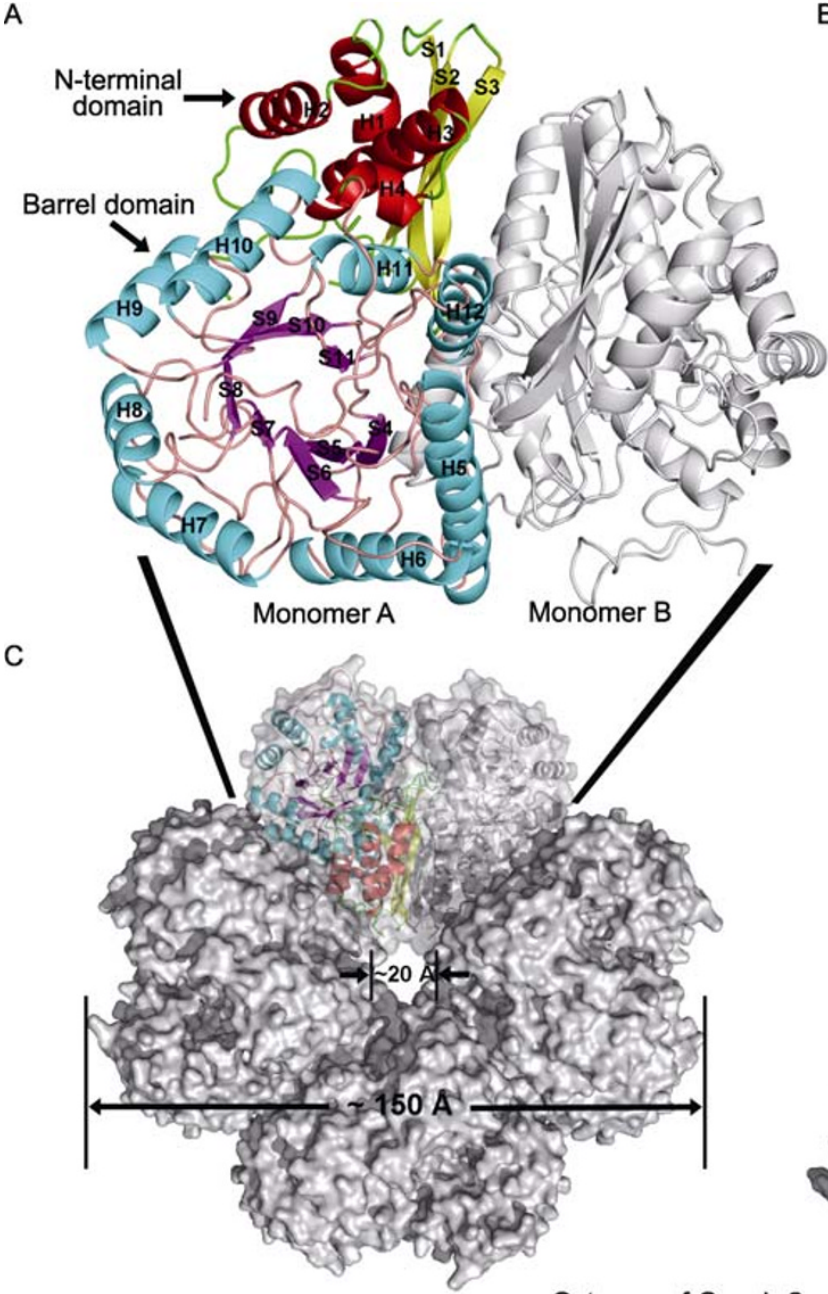

B

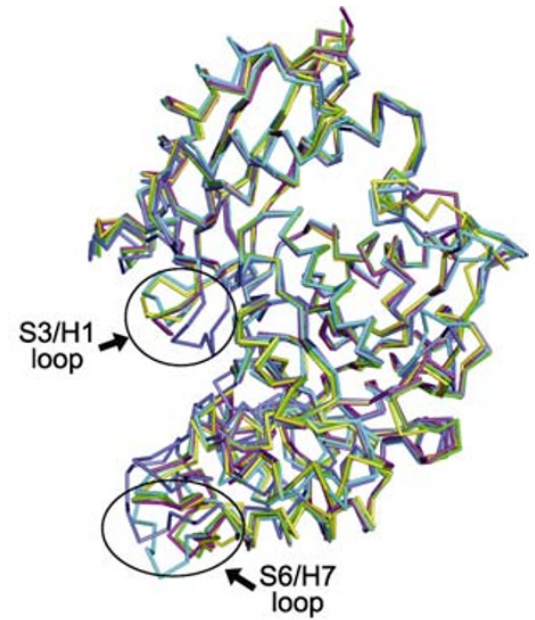

Octamer of $S$. suis 2 enolase

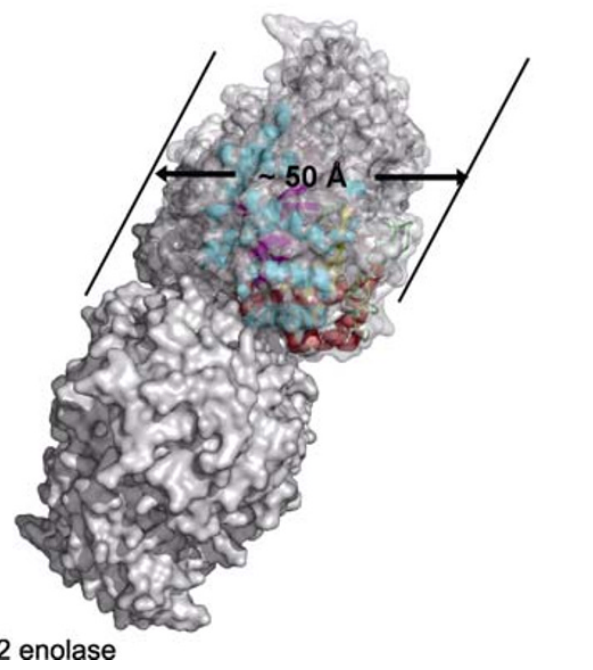

Figure 2. An octameric structure of SS2 enolase in crystal. (A) The overall structure of SS2 enolase. The two enolase monomers (monomer A and B) within the asymmetric unit, which form a tight homo-dimer, are shown in cartoon representation. Each monomer could be further divided into an $\mathrm{N}$-terminal domain and a $\mathrm{C}$-terminal barrel domain, which are highlighted in red/yellow/green and cyan/magenta/orange modes, respectively. The secondary structural elements are labeled. (B) A ribbon shown highlighting the overall structural conservation among enolases of representative species including $S$. suis (green), $S$. pneumoniae (magenta), E. coli (yellow), yeast (cyan) and human (blue). The two loop regions which are of conformational variance are marked with black circles and labeled. (C) The octameric structure of SS2 enolase generated by symmetry operation. Four "heart"-like enolase dimers assembled into a ring-shaped octamer entity. The size of the operation-yielded octamer is indicated.

have occasionally been reported (Schurig et al., 1995; Ehinger et al., 2004; Karbassi et al., 2010). Our previous study on SS2 enolase indicates an octamer enzyme in solution by gel filtration analysis (Feng et al., 2009). Consistent with this, despite that the asymmetric unit of our structure contains only an enolase dimer, simple symmetry operations could yield a similar octamer as observed in the enolase structure of S. pneumonia (Ehinger et al., 2004). As shown in Fig. 2C, each "heart"-like enolase dimer interacts extensively with the neighboring dimer unit and four of such dimers assemble into a ring-shaped octamer entity. The size of the operation-yielded octamer is $\sim 150 \AA$ in diameter and $\sim 50 \AA$ in thickness (Fig. 2C). A small tunnel, which is only $\sim 20 \AA$ in diameter, was observed in the center of the octamer (Fig. 2C).

We then set out to further investigate the oligomeric state of SS2 enolase in solution. The purified protein was analyzed by ultracentrifugation assay. As expected, the sedimentation profile reveals only a single protein species, the molecular weight of which is calculated to be $370 \mathrm{kDa}$ (Fig. $3 \mathrm{~A}$ ). This result clearly demonstrates that SS2 enolase exclusively exits as an octamer in solution. Therefore, we provided both structural and biophysical evidence of an octamer of enolase in SS2. 

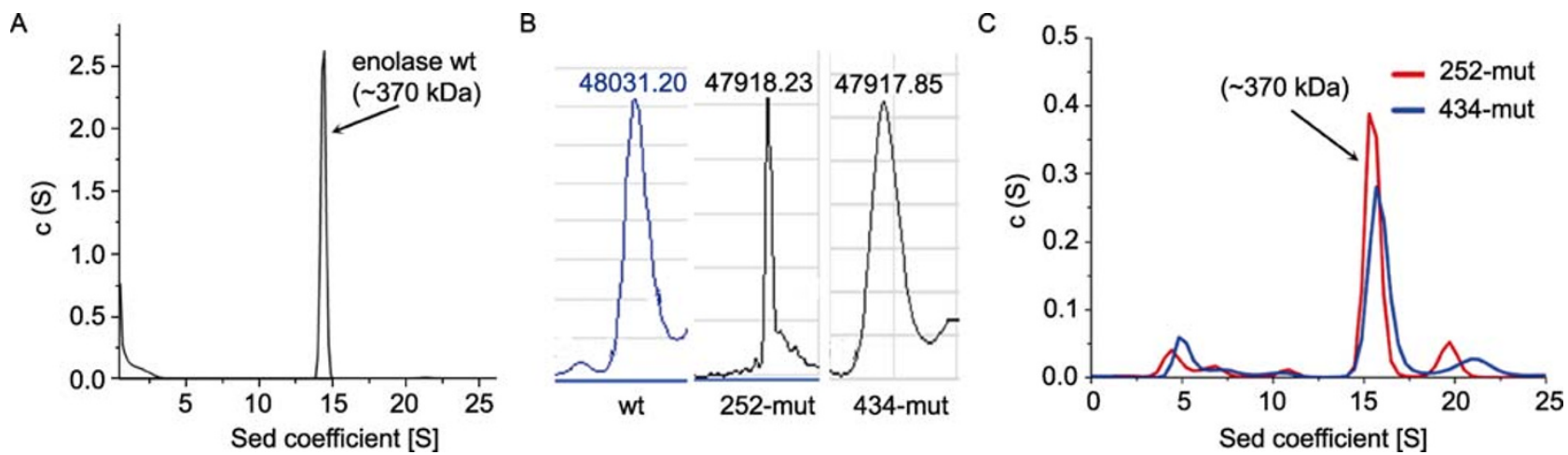

Figure 3. SS2 enolase exists as an octamer in solution. (A) The purified wt SS2 enolase protein is analyzed by ultracentrifugation which reveals a single protein species of about $370 \mathrm{kDa}$ in molecular weight. The sedimentation profile is shown. (B) Mass-spectrometric analysis of the recombinant SS2 enolase for the wt, the 252-mut and 434-mut proteins. The determined molecular weight for the indicated protein is highlighted. (C) The mutant proteins also assembled into octamers in solution. The sedimentation profiles are shown.

\section{Extended dimerization and octamerization interfaces}

The identification of a stable enolase octamer both in crystal and in solution prompted us to further explore the contacting interfaces mediating octamer assembly. The symmetry-related octamer formation within the structure indicates two types of oligomerization interfaces: one holds the two monomers together (from monomer to dimer), and the other fastens/clinches the neighboring dimers (from dimer to octamer) (Fig. 4A).

We first scrutinized those residues involved in dimer formation. As shown in Fig. 4B, the two enolase molecules pack against each other via an extensive interface, burying a total of $1717 \AA^{2}$ surface area. This interface, which is composed of 26 amino acids in each monomer, can roughly be further divided into three continuous surface patches. A bunch of hydrophilic residues arranged into two circles, each surrounding a small hydrophobic center, to form dimer inter-patches 1 and 2 (Fig. 4B). The first inter-patch mainly localizes in the $\mathrm{N}$-terminal domain, with a small number of residues (e.g., Glu378, Thr402, Asp403) from the enolase $\mathrm{C}$-terminal barrel. While the second patch is exclusively located in the barrel domain of SS2 enolase. Since the two molecules in the dimer are 2-fold axis related (see results above), those residues involved in each of the two inter-patches are almost identical in the respective monomers. Small differences lie in 4 amino acids including Asn18, Arg180, Ser57 and Arg401. The former two residues are part of the inter-patches only in monomer $A$, while the latter two are solely observed in those of monomer B (Fig. 4B). Overall, inter-patch 1 of one molecule contacts mainly inter-patch 2 of the other, and vice versa, to form a network of strong $\mathrm{H}$-bonds and hydrophobic interactions, tying the two monomers together. Additional strengthening force stabilizing the enolase dimer comes from the interaction be- tween a 3-residue apolar patch (inter-patch 3) in the respective molecules. These three amino acids, including Val199, Ala201 and Val202, localize between the inter-patches 1 and 2, and further clinch the enolase monomers via hydrophobic contacts (Fig. 4B). It is also noteworthy that unlike a mainly hydrophobic interface commonly observed in protein oligomerization, the enolase dimer interactions are predominantly polar contacts with 18 interface residues (out of 26 ) being amino acids of hydrophilic characters.

The octameric interface is on the opposite side of that of the dimer (Fig. 4A), burying a surface area of about $1227 \AA^{2}$ in the two neighboring enolase monomers. A total of 15 residues were involved in this interface, forming two separate surface patches. The first patch (octamer inter-patch 1) is much more extended with 12 amino acids, and is exactly the same in the respective enolase monomers. Six polar residues including GIn88, Lys140, Glu356, Glu359, Lys362 and Asn390 are aligned almost into a line, bordering the octameric interface on one side. The intimate inter-molecule contacts among these residues form a strong hydrogen bond network. In addition, being a highly hydrophobic amino acid itself, Phe137 also participates in the polar interactions by forming an $\mathrm{H}$-bond using its main-chain oxygen with GIn88 of the neighboring molecule. The apolar portion of octamer inter-patch 1 is composed of residues Ala89, Leu134, Phe137, Leu351, Phe355 and Val421, forming the hydrophobic centre of the octameric interface. In contrast to patch1, the octamer inter-patch 2 only involves three amino acids and exhibits difference in residue compositions in the interacting enolase entities. This patch contributes to the octamer assembly mainly through an electrostatic attraction between Arg85 of one monomer and Glu127 of the other, as well as the hydrophobic interactions among Leu126 and Leu128 of the contacting molecules (Fig. 4C). 


\section{SS2 enolase octamer potently binds to plasminogen}

Several studies have demonstrated that enolase could bind to plasminogen (Andronicos et al., 1997; Bergmann et al., 2001; Agarwal et al., 2008; Candela et al., 2009). Our previous study on SS2 enolase has indicated its role in the adherence of SS2 to epidermal cells such as Hep-2 (Feng et al., 2009). Therefore, we used an in vitro assay to investigate the binding characteristics of SS2 enolase to human plasminogen. The wt enolase octamer of gradient concentrations was passed over the immobilized plasminogen in SPR experiments. The potent binding between these two proteins is demonstrated, as only $31.25 \mathrm{nmol} / \mathrm{L}$ of enolase could yield obvious reflection signals (Fig. 5A). The Biacore ${ }^{\circledR}$ profile is similar to that observed for antigen and antibody with fast association but slow dissociation kinetics. Based on previous reports (Derbise et al., 2004; Ehinger et al., 2004; Cork et al., 2009), there should be multiple plasminogen binding sites in the enolase octamer. Therefore, no appropriate model could be used for the calculation of the dissociation constant $\left(\mathrm{K}_{\mathrm{d}}\right)$. Unable to determine the exact binding affinity, the Biacore ${ }^{\circledR}$ result clearly shows that the enolase octamer of $S$. suis 2 is active in plasminogen binding.

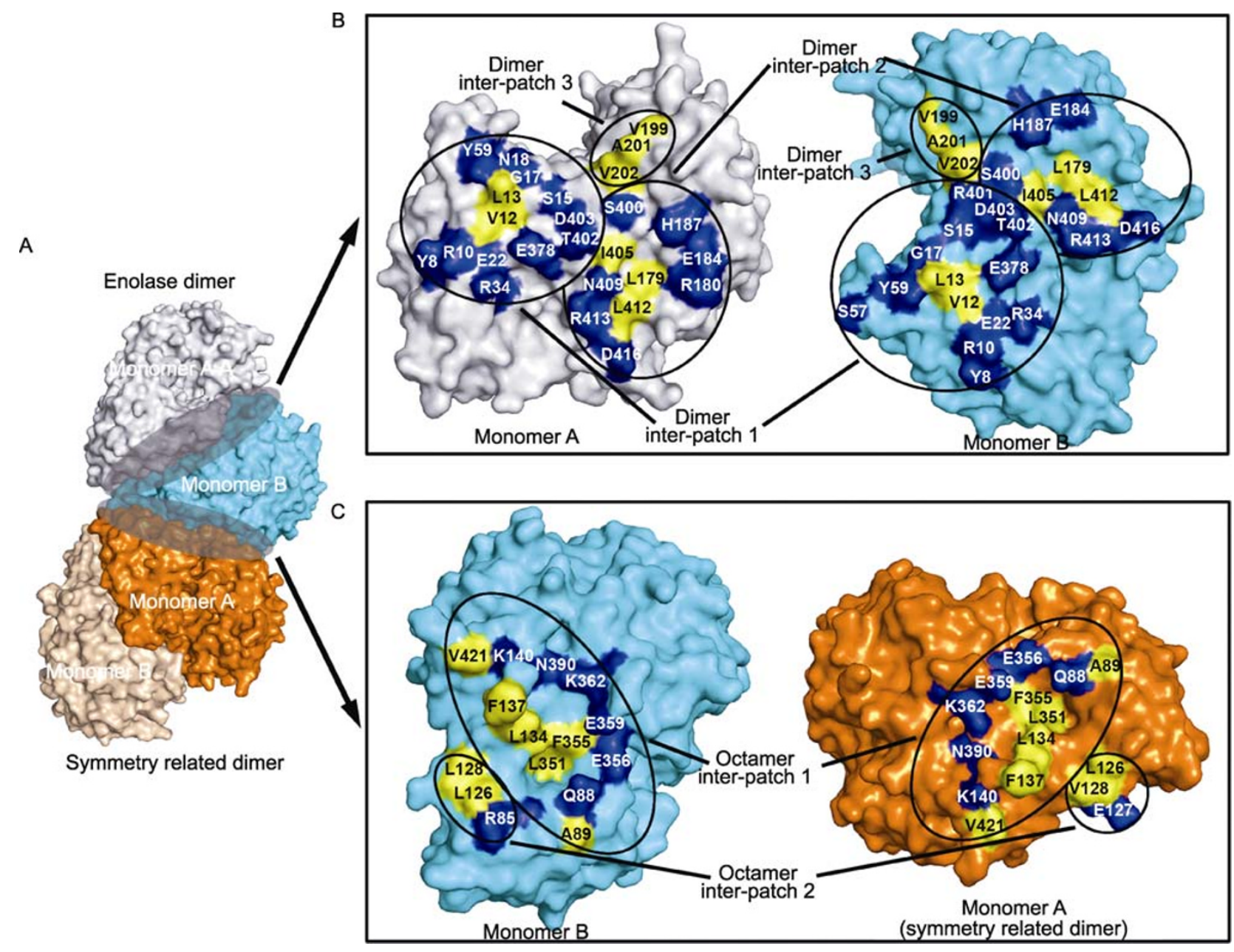

Figure 4. Extended dimerization and octamerization interfaces in SS2 enolase octamer. (A) A surface presentation showing the two types of interfaces (dimerization and octamerization) identified in the SS2 enolase octamer. Two enolase dimers are shown with the four monomers colored in light gray, cyan, orange and wheat, respectively. The representative interfaces, which are characterized in detail in (B) and (C), are shaded for highlight. (B and C) Detailed views of the dimer and octamer interfaces in the SS2 enolase structure. A total of 26 amino acids (in each monomer) are involved in the dimer formation which are arranged into three continuous surface patches (dimer inter-patch 1, 2 and 3), whereas 15 residues are shown to mediate the octamer formation, forming two separate parts (octamer inter-patch1 and 2). Each inter-patch is indicated, and those residues referred to in the text are labeled. The hydrophilic and hydrophobic interactions are highlighted in blue and yellow colors, respectively. (B) The dimerization interface. (C) The octamerization interface. 
A

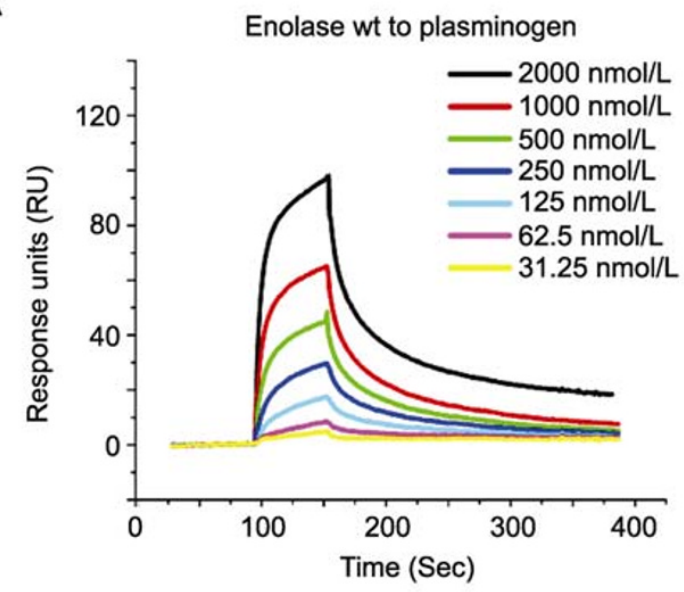

C

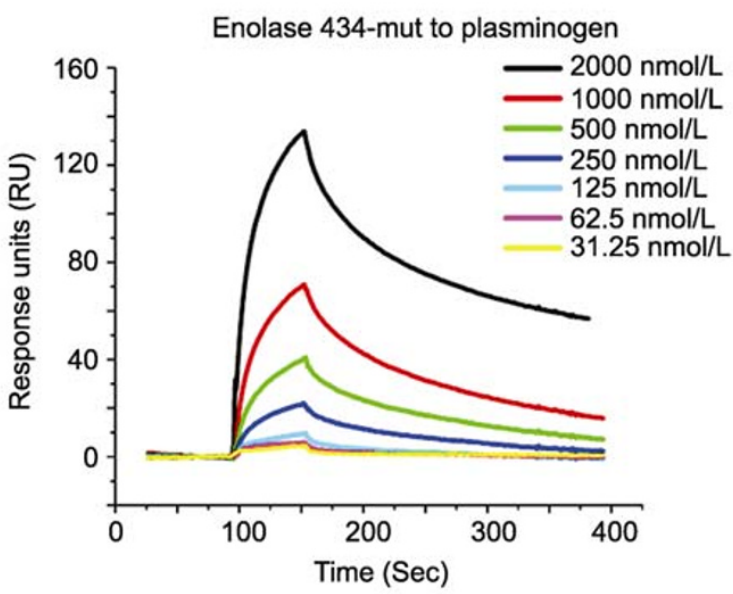

B

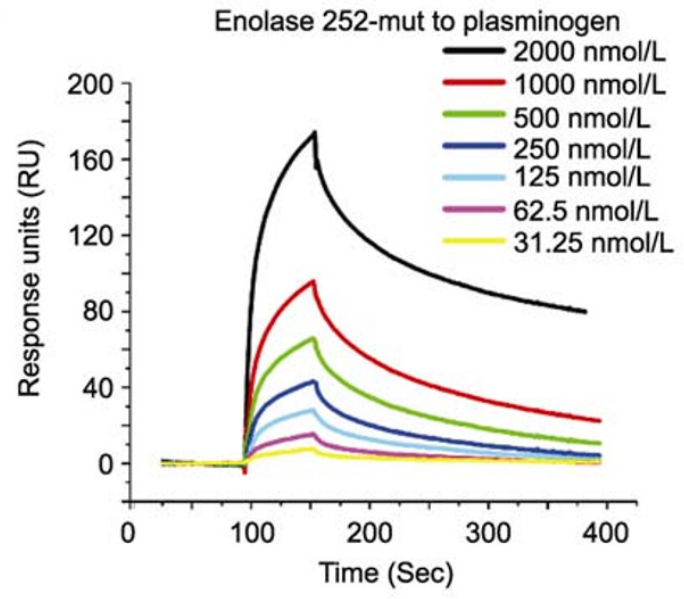

D

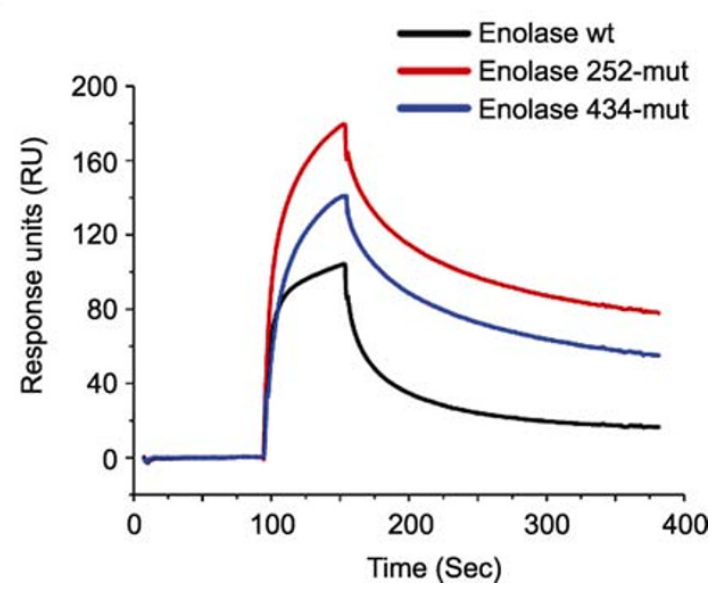

Figure 5. Binding of the SS2 enolase to plasminogen. (A, B and C) Gradient concentrations of SS2 enolase (wt, 252-mut and 434-mut) ranging from $31.25 \mathrm{nmol} / \mathrm{L}$ to $2000 \mathrm{nmol} / \mathrm{L}$ flow through immobilized plasminogen in an SPR assay. The binding profiles are shown. (A) wt enolase. (B) The 252-mut enzyme. (C) The 434-mut enzyme. (D) An integrated view comparing the binding response units for the indicated enolase proteins $(2000 \mathrm{nmol} / \mathrm{L})$.

\section{Plasminogen binding sites in SS2 enolase are different from those of other Streptococcus spp}

The plasminogen binding sites have been mapped to the "FYDKERKVY" loop and the C-terminal double lysine residues in the enolases from Streptococcus pyogenes and Streptococcus pneumoniae (Bergmann et al., 2003; Derbise et al., 2004; Cork et al., 2009). These two sequence motifs are both highly conserved in the enolase of SS2 (Fig. 1). The first motif is part of the $\mathrm{S} 6 / \mathrm{H} 7$ intervening loop and is completely solvent exposed in the octamer structure of SS2 enolase (Fig. 6). While without electron densities for Lys 434 and Lys 435 (also see the results above), the steric positions for the residues directly preceding these two distal C-terminal amino acids could be clearly traced. Therefore, the second motif locates near the octameric interface between the neighboring enolase dimers. There might be some restriction (from the vicinity molecule) over the accessibility of this second motif by the potential ligand. Nevertheless, this restriction is rather limited, leaving enough steric space where some inter-chain contacts could occur (Fig. 6).

The structural analysis indicates the feasibility of these two sequence motifs as the potential plasminogen binding sites in SS2 enolase. We therefore did mutation work of the two lysine residues in either motif (termed 252-mut for alanine replacement of K252 and K255, and 434-mut for that of K434 and K435) and tested the binding of the mutant enolase proteins to immobilized plasminogen by SPR. Successful substitution of the lysine residues by alanine was confirmed by mass spectrometry (Fig. 3B). As the mutated sites are sterically away from both the dimeric and the octameric interfaces, the mutant proteins also assembled into octamers in solution (Fig. $3 \mathrm{C}$ ). Nevertheless, to our great surprise, the two enolase mutants still potently bind to plasminogen (Fig. 5B and 5C), 
A

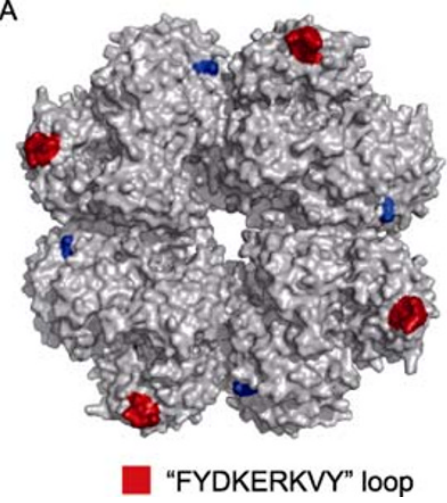

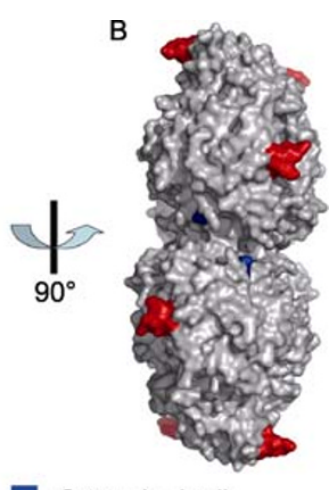

C-terminal tail

Figure 6. The putative plasminogen binding sites in SS2 enolase deduced from other Streptococcus spp. Two motifs including the "FYDKERKVY" loop and the distal C-terminal double lysine residues have been indicated in the plasminogen binding of $S$. pyogenes enolase. Both motifs are highly conserved in the SS2 enolase and ligand accessible. Yet it is confirmed in our study that these sites might not be the main plasminogen-binding sites. The SS2 enolase octamer is shown in surface presentation with the two putative binding motifs highlighted in red and blue, respectively. (A) Front view of the octamer structure. (B) Side view of the octamer structure.

as observed for the wt protein. In contrast to those reported for the enolases from S. pyogenes and S. pneumoniae, none of these mutations decrease the SS2 enolase binding to human plasminogen (Fig. 5D).

\section{DISCUSSION}

Enolase is a functionally important enzyme in both prokaryotic and eukaryotic organisms (Gerlt et al., 2012). The enolase from SS2 is evolutionarily conserved in terms of its metal coordination and 2-phosphoglycerate (2-PG) hydrolysis activity, which we have already fully characterized in our previous study (Feng et al., 2009). In addition to its role in the carbohydrate metabolism, the enzyme has also been implicated in the SS2 virulence by facilitating the adherence of bacteria to the host cells (Feng et al., 2009; Zhang et al., 2009). Yet the exact mechanism underlying the virulence contribution of SS2 enolase has not been illustrated. In this study, we confirmed the plasminogen binding capability of SS2 enolase via surface plasmon resonance, and found similar binding characteristics to those of a previous report (Esgleas et al., 2008). We believe this binding property is involved in the much more effective host adherence of the wild-type $S$. suis strain over the enolase knock-out mutant. This provides an opportunity for designing antibodies against plasminogen, thus preventing the pathogenic bacterial migration across the infected cellular membrane and therefore controlling $S$. suis infections in humans. This raises an op-

portunity that could warrant further study in the future.

The oligomerization state of enolase has always been an interesting issue. Most enolases are dimers in solution (Brown et al., 1998) but enolase octamers have not been rare either (Schurig et al., 1995; Ehinger et al., 2004; Karbassi et al., 2010). A previous study proposed that the length of the loop connecting $\mathrm{H} 4$ helix and S4 strand is possibly a key factor determining enolase octamerization (Brown et al., 1998). A long H4/S4 loop corresponds to enolase dimer, while a short loop could lead to the octamer formation. The assay data of this work supports this viewpoint as SS2 enolase contains a short loop in this position and indeed forms the octamer both in crystal and in solution. In the structure of SS2 enolase, two residues in this loop (Phe137 and Lys140) are part of the octamer inter-patch 1. Apparently, the H4/S4 loop is indispensably involved in the octamer interfaces of enolases from S. suis, S. pneumoniae, S. pyogenes, etc. all of which have been demonstrated to be octameric. Extra residues in this loop could result in a strong steric hindrance and thereby prevent enolase dimers from approaching into higher oligomeric forms, which appears to be the case for human, Yeast, E. coli, etc. (Stec and Lebioda, 1990; Kuhnel and Luisi, 2001; Kang et al., 2008). With great efforts, we failed to obtain any octameric forms in the structure for enolases from human, Yeast and $E$. coli by symmetry operation.

In our previous study, we showed that purified SS2 enolase is catalytically capable (Feng et al., 2009). In this study, we provided solid structural and biophysical evidence that the enolase enzyme exists exclusively in the octameric form. We further demonstrated that this octamer is active in plasminogen binding. These results indicate that an octamer is the biological unit of SS2 enolase at least in vitro and quite possibly in vivo as well.

It remains an unsettled issue as to the plasminogen binding sites in enolase. Previous studies have proposed that the "FYDKERKVY" loop and the C-terminal double Lysine residues are involved in the interaction of enolase with plasminogen. Nevertheless, discrepancies remain. The work on the $S$. pyogenes enzyme proved the indispensability of the C-terminal lysine residues in plasminogen binding. But, the mutant with lysine mutations in the "FYDKERKVY" loop remains binding-capable to plasminogen in a ligand-blot assay (Cork et al., 2009). As for the S. pneumoniae enolase, it is the loop-mutation rather than the terminal-lysine substitutions that almost completely disrupt the enolase/plasminogen interactions (Bergmann et al., 2003). In our study, we unexpectedly found that the SS2 enolase with lysine mutations in either of the two sequence motifs bound to human plasminogen as potent as the wild-type enzyme. Noted that the enolases from S. suis, S. pygenes and S. pneumonia only differ in several amino acids in sequences (Fig. 1), it is unlikely that the three enzymes utilize quite different surface residues for plasminogen interactions. We therefore believe 
that the observed discrepancies must indicate the involvement of some other, yet unidentified but likely even more important, structural elements in the plasminogen-binding of enolase. Further studies are needed to settle the issue.

\section{MATERIALS AND METHODS}

\section{Protein production and crystallization}

The gene (gi: 146317663) coding for the enolase was amplified from the genomic DNA extract of SS2 strain 05ZYH33 by polymerase chain reaction (PCR) using the following primers: $5^{\prime}$-GGAATTCCATATGTCAATTATTACTG-3' and 5'-CCGCTCGAGTTTTTTCAAGTTGTAG-3', which contained Ndel and Xhol restriction sites, respectively. The PCR product was then cloned into pET21a vector (Novagen) to yield the expressing plasmid which encodes a protein with a purification tag (LEHHHHHH) at the end of the full-length SS2 enolase.

For site-directed mutagenesis, the plasmid encoding the wild type (wt) enolase was used as the template. Using the following primer pairs, the mutant constructs, which encode enolase K252A/K255A mutant (termed 252-mut) and K434A/K435A mutant (termed 434-mut), were successfully obtained using the Phusion Site Mutagenesis Kit (NEB). The identities of all the DNA constructs were confirmed by sequencing.

252-mutF: 5'-TTCTACGACgcgGAACGTgcgGTTTACGAC-3';

252-mutR: 5'-GTCGTAAACcgcACGTTCcgcGTCGTAGAA-3'; 434-mutF: 5'-TTGAACTCATTCTACAACTTGgcggcgCTCGAG-3'; 434-mutR: 5'-CTCGAGcgccgcCAAGTTGTAGAATGAGTTCAA-3'.

The preparation of the SS2 enolase (wt, 252-mut or 434-mut) followed a previous purification procedure for soluble His-tagged proteins (Lu et al., 2011b). The plasmid carrying the correct gene was introduced into $E$. coli BL21 (DE3). Proteins were expressed in $E$. coli $\mathrm{BL} 21$ cells grown at $37^{\circ} \mathrm{C}$ in $\mathrm{LB}$ medium to an $\mathrm{OD}_{600}$ of $0.6-0.8$, induced with $0.1 \mathrm{mmol} / \mathrm{L}$ of isopropyl b-D-thiogalactopyranoside (IPTG) and then incubated for $\sim 4 \mathrm{~h}$ at $37^{\circ} \mathrm{C}$. Cells were harvested by centrifugation and lysed using sonication in $20 \mathrm{mmol} / \mathrm{L}$ Tris- $\mathrm{HCl}(\mathrm{pH} 8.0)$ and $50 \mathrm{mmol} / \mathrm{L} \mathrm{NaCl}$. Soluble protein was purified by nickel-affinity chromatography followed by size-exclusion chromatography on a superdex200 ${ }^{\circledR}$ FPLC column (Amersham Biosciences). Protein-containing fractions were analysed by SDS-PAGE. The purified protein was then concentrated to $25 \mathrm{mg} / \mathrm{mL}$ using an Amicon ultra- 15 filter (Millipore). The identity of the individual enolase protein (wt, 252-mut and 434-mut) was further verified by mass-spectrometry using the Agilent 6530 Accurate-Mass Quadrupole Time-of-Flight (Q-TOF) LC-MS system (Agilent).

\section{Crystallization, data collection, structure determination and refinement}

The initial crystallization trials were set up with the commercial available Hampton Kits using the hanging-drop vapor diffusion method. Equal volumes $(1 \mu \mathrm{L})$ of the wt protein and the reservoir solutions were mixed and crystals were grown at $277 \mathrm{~K}$. Those conditions that could yield protein crystals were further optimized. Well-diffracted crystals were finally obtained in a condition consisting of $0.1 \mathrm{~mol} / \mathrm{L}$
MES (pH 6.5) and 10\% (w/v) PEG 20,000.

For data collection, a single crystal was first immersed into a cryo-protectant consisting of $17 \%$ glycerol and $83 \%$ reservoir solution for $\sim 30 \mathrm{~s}$. The crystal was then mounted on a nylon loop and flash-cooled in a nitrogen gas stream at $100 \mathrm{~K}$. X-ray data were in-house collected using a Rigaku rotating copper anode equipped with an R-axis image plate. The diffraction images were processed with HKL2000 (Otwinowski et al., 1997). The initial model of the SS2 enolase was built by molecular replacement using the program MOLREP (Vagin and Teplyakov, 1997) with the structure of its homologous protein from $S$. pneumoniae as a search model (PDB code 1W6T). The structure was completed using alternate cycles of manual building in COOT (Emsley and Cowtan, 2004) and refinement using REFMAC5 (Murshudov et al., 1997) as implemented in the CCP4 suite (collaborative., 1994) and Phenix.refine (Adams et al., 2010). The stereo-chemical quality of the final model was assessed by Procheck (Laskowski, 1993). The final coordinates and its related structural factors were deposited into the RCSB Protein Data Bank with the accession number of 4EWJ. The final statistics for data collection and structural refinement are summarized in Table 1.

\section{Surface plasmon resonance (SPR) measurements}

The binding kinetics between the purified SS2 enolase protein (wt, 252-mut or 434-mut) and human plasminogen (purchased from Sigma) was analyzed at $25^{\circ} \mathrm{C}$ on a BIAcore ${ }^{\circledR} 3000$ with CM5 chips (GE Healthcare). The HBS-EP buffer (10 mmol/L HEPES, 150 $\mathrm{mmol} / \mathrm{L} \mathrm{NaCl}, 3 \mathrm{mmol} / \mathrm{L}$ EDTA, $0.005 \%$ Tween-20) was used for all measurements. We used the blank channel as negative control. About 2400 response units (RU) of plasminogen were immobilized on the chip, followed by blockade with ethylenediamine. When the data collection was finished in each cycle, the sensor surface was regenerated with $10 \mathrm{mmol} / \mathrm{L} \mathrm{NaOH}$. A series of concentrations ranging from $31 \mathrm{nmol} / \mathrm{L}$ to $2 \mu \mathrm{mol} / \mathrm{L}$ were used in the experiment.

\section{Analytical ultracentrifugation}

The sedimentation experiment was performed on a Beckman XL-A analytical ultracentrifuge (Fullerton, CA). The enolase sample (wt, 252-mut or 434-mut) was used at an initial concentration of $A_{280}=0.8$ absorbance units in a buffer consisting of $50 \mathrm{mmol} / \mathrm{L} \mathrm{Tris} / \mathrm{HCl}(\mathrm{pH} 7.4)$, $1 \mathrm{mmol} / \mathrm{L} \mathrm{Mg}(\mathrm{OAc})_{2}, 0.1 \mathrm{mmol} / \mathrm{L}$ EDTA. The protein sample $(400 \mu \mathrm{L})$ and the reference solution $(400 \mu \mathrm{L} 50 \mathrm{mmol} / \mathrm{L}$ Tris/ $\mathrm{HCl}(\mathrm{pH} 7.4), 1$ $\mathrm{mmol} / \mathrm{L} \mathrm{Mg}(\mathrm{OAc})_{2}, 0.1 \mathrm{mmol} / \mathrm{L}$ EDTA) were loaded into a conventional double-sector quartz cell and mounted in a Beckman Coulter An-60 Ti 4-hole rotor. The sedimentation velocity was recorded at a speed of $35,000 \mathrm{rpm}$ at $20^{\circ} \mathrm{C}$. The profiles were measured by UV absorbance at $280 \mathrm{~nm}$ in a continuous mode with time intervals of $480 \mathrm{~s}$. The recorded scans for the various time points were collected and analyzed using the $\mathrm{c}(\mathrm{s})$ continuous size distribution model with the SEDFIT program Version11.3b. Molecular weights were estimated using the $c(M)$ model after fitting the frictional ratio $(f / f O)$.

\section{ACKNOWLEDGEMENTS}

We thank Dr. Qiangmin Zhang and Mr. Joel Haywood for help. The 
technical support from Zheng Fan was acknowledged. This work is supported by National Natural Science Foundation of China (NSFC). GFG is a leading principal investigator of the NSFC Innovative Research Group (Grant No. 81021003).

\section{ABBREVIATIONS}

2-PG, 2-phosphoglycerate; IPTG, isopropyl b-D-thiogalactopyranoside; $\mathrm{PI}$, pathogenecity island; SS2, Streptococcus suis serotype 2; STSS, streptococcal toxic shock syndrome

\section{REFERENCES}

Adams, P.D., Afonine, P.V., Bunkoczi, G., Chen, V.B., Davis, I.W., Echols, N., Headd, J.J., Hung, L.W., Kapral, G.J., Grosse-Kunstleve, R.W., et al. (2010). PHENIX: a comprehensive Python-based system for macromolecular structure solution. Acta Crystallogr D Biol Crystallogr 66, 213-221.

Agarwal, S., Kulshreshtha, P., Mukku, D.B., and Bhatnagar, R. (2008). alpha-Enolase binds to human plasminogen on the surface of Bacillus anthracis. Bba-Proteins Proteom 1784, 986-994.

Andronicos, N.M., Ranson, M., Bognacki, J., and Baker, M.S. (1997). The human ENO1 gene product (recombinant human alpha-enolase) displays characteristics required for a plasminogen binding protein. Bba-Protein Struct M 1337, 27-39.

Babbitt, P.C., Hasson, M.S., Wedekind, J.E., Palmer, D.R., Barrett, W.C., Reed, G.H., Rayment, I., Ringe, D., Kenyon, G.L., and Gerlt, J.A. (1996). The enolase superfamily: a general strategy for enzyme-catalyzed abstraction of the alpha-protons of carboxylic acids. Biochemistry-US 35, 16489-16501.

Banner, D.W., Bloomer, A.C., Petsko, G.A., Phillips, D.C., Pogson, C.I., Wilson, I.A., Corran, P.H., Furth, A.J., Milman, J.D., Offord, R.E., et al. (1975). Structure of chicken muscle triose phosphate isomerase determined crystallographically at 2.5 angstrom resolution using amino acid sequence data. Nature 255, 609-614.

Baums, C.G., Kaim, U., Fulde, M., Ramachandran, G., Goethe, R., and Valentin-Weigand, P. (2006). Identification of a novel virulence determinant with serum opacification activity in Streptococcus suis. Infect Immun 74, 6154-6162.

Bergmann, S., Rohde, M., Chhatwal, G.S., and Hammerschmidt, S. (2001). alpha-Enolase of Streptococcus pneumoniae is a plasmin(ogen)-binding protein displayed on the bacterial cell surface. Mol Microbiol 40, 1273-1287.

Bergmann, S., Wild, D., Diekmann, O., Frank, R., Bracht, D., Chhatwal, G.S., and Hammerschmidt, S. (2003). Identification of a novel plasmin(ogen)-binding motif in surface displayed alpha-enolase of Streptococcus pneumoniae. Mol Microbiol 49, 411-423.

Brown, C.K., Kuhlman, P.L., Mattingly, S., Slates, K., Calie, P.J., and Farrar, W.W. (1998). A model of the quaternary structure of enolases, based on structural and evolutionary analysis of the octameric enolase from Bacillus subtilis. J Protein Chem 17, 855-866.

Candela, M., Biagi, E., Centanni, M., Turroni, S., Vici, M., Musiani, F., Vitali, B., Bergmann, S., Hammerschmidt, S., and Brigidi, P. (2009). Bifidobacterial enolase, a cell surface receptor for human plasminogen involved in the interaction with the host.
Microbiol-Sgm 155, 3294-3303.

Chandran, V., and Luisi, B.F. (2006). Recognition of enolase in the Escherichia coli RNA degradosome. J Mol Biol 358, 8-15.

Chen, C., Tang, J., Dong, W., Wang, C., Feng, Y., Wang, J., Zheng, F., Pan, X., Liu, D., Li, M., et al. (2007). A glimpse of streptococcal toxic shock syndrome from comparative genomics of $\mathrm{S}$. suis 2 Chinese isolates. Plos One 2, e315.

collaborative. (1994). The CCP4 suite:programs for protein crystallography. Acta Crystallogr D Biol crystallography 50, 760-763.

Cork, A.J., Jergic, S., Hammerschmidt, S., Kobe, B., Pancholi, V., Benesch, J.L., Robinson, C.V., Dixon, N.E., Aquilina, J.A., and Walker, M.J. (2009). Defining the structural basis of human plasminogen binding by streptococcal surface enolase. J Biol Chem 284, 17129-17137.

Derbise, A., Song, Y.P., Parikh, S., Fischetti, V.A., and Pancholi, V. (2004). Role of the C-terminal lysine residues of streptococcal surface enolase in Glu- and Lys-plasminogen-binding activities of group A streptococci. Infect Immun 72, 94-105.

Ehinger, S., Schubert, W.D., Bergmann, S., Hammerschmidt, S., and Heinz, D.W. (2004). Plasmin(ogen)-binding alpha-enolase from Streptococcus pneumoniae: Crystal structure and evaluation of plasmin(ogen)-binding sites. J Mol Biol 343, 997-1005.

Emsley, P., and Cowtan, K. (2004). Coot: model-building tools for molecular graphics. Acta Crystallogr D Biol Crystallogr 60, 2126-2132.

Esgleas, M., Li, Y., Hancock, M.A., Harel, J., Dubreuil, J.D., and Gottschalk, M. (2008). Isolation and characterization of alpha-enolase, a novel fibronectin-binding protein from Streptococcus suis. Microbiology 154, 2668-2679.

Feng, Y., Pan, X., Sun, W., Wang, C., Zhang, H., Li, X., Ma, Y., Shao, Z., Ge, J., Zheng, F., et al. (2009). Streptococcus suis enolase functions as a protective antigen displayed on the bacterial cell surface. J Infect Dis 200, 1583-1592.

Feng, Y., Zhang, H., Ma, Y., and Gao, G.F. (2010). Uncovering newly emerging variants of Streptococcus suis, an important zoonotic agent. Trends Microbiol 18, 124-131.

Gerlt, J.A., Babbitt, P.C., Jacobson, M.P., and Almo, S.C. (2012). Divergent evolution in enolase superfamily: strategies for assigning functions. J Biol Chem 287, 29-34.

Gerlt, J.A., Babbitt, P.C., and Rayment, I. (2005). Divergent evolution in the enolase superfamily: the interplay of mechanism and specificity. Arch Biochem Biophys 433, 59-70.

Guo, Y., Wang, J., Niu, G., Shui, W., Sun, Y., Zhou, H., Zhang, Y., Yang, C., Lou, Z., and Rao, Z. (2011). A structural view of the antibiotic degradation enzyme NDM-1 from a superbug. Protein Cell 2, 384-394.

Han, H., Liu, C., Wang, Q., Xuan, C., Zheng, B., Tang, J., Yan, J., Zhang, J., Li, M., Cheng, H., et al. (2012). The two-component system Ihk/Irr contributes to the virulence of Streptococcus suis serotype 2 strain $05 \mathrm{ZYH} 33$ through alteration of the bacterial cell metabolism. Microbiology. (In Press)

Holm, L., and Sander, C. (1996). Mapping the protein universe. Science 273, 595-602.

Hosaka, T., Meguro, T., Yamato, I., and Shirakihara, Y. (2003). Crystal structure of Enterococcus hirae enolase at $2.8 \mathrm{~A}$ resolution. J Biochem 133, 817-823. 
Jones, M.N., and Holt, R.G. (2007). Cloning and characterization of an alpha-enolase of the oral pathogen Streptococcus mutans that binds human plasminogen. Biochem Bioph Res Co 364, 924-929.

Kang, H.J., Jung, S.K., Kim, S.J., and Chung, S.J. (2008). Structure of human alpha-enolase (hENO1), a multifunctional glycolytic enzyme. Acta Crystallogr D Biol Crystallogr 64, 651-657.

Karbassi, F., Quiros, V., Pancholi, V., and Kornblatt, M.J. (2010). Dissociation of the octameric enolase from $\mathrm{S}$. pyogenes--one interface stabilizes another. Plos One 5, e8810.

Kuhnel, K., and Luisi, B.F. (2001). Crystal structure of the Escherichia coli RNA degradosome component enolase. J Mol Biol 313, 583-592.

Laskowski, R.A., MacArthur, M. W., Moss, D. S., \& Thorton, J. M (1993). PROCHECK: a program to check the stereochemical quality of protein structures. J Appl Crystallogr 26, 283-291.

Li, M., Shen, X., Yan, J., Han, H., Zheng, B., Liu, D., Cheng, H., Zhao, Y., Rao, X., Wang, C., et al. (2011). Gl-type T4SS-mediated horizontal transfer of the $89 \mathrm{~K}$ pathogenicity island in epidemic Streptococcus suis serotype 2. Mol Microbiol 79, 1670-1683.

Li, M., Wang, C., Feng, Y., Pan, X., Cheng, G., Wang, J., Ge, J., Zheng, F., Cao, M., Dong, Y., et al. (2008). SalK/SalR, a two-component signal transduction system, is essential for full virulence of highly invasive Streptococcus suis serotype 2. Plos One 3, e2080.

Lu, G., Qi, J., Gao, F., Yan, J., Tang, J., and Gao, G.F. (2011a). A novel "open-form" structure of sortaseC from Streptococcus suis. Proteins 79, 2764-2769.

Lu, G., Zhang, J., Li, Y., Li, Z., Zhang, N., Xu, X., Wang, T., Guan, Z., Gao, G.F., and Yan, J. (2011b). hNUDT16: a universal decapping enzyme for small nucleolar RNA and cytoplasmic mRNA. Protein Cell 2, 64-73.

Ma, Y., Feng, Y., Liu, D., and Gao, G.F. (2009). Avian influenza virus, Streptococcus suis serotype 2, severe acute respiratory syndrome-coronavirus and beyond: molecular epidemiology, ecology and the situation in China. Philos Trans R Soc Lond B Biol Sci 364, 2725-2737.

Murshudov, G.N., Vagin, A.A., and Dodson, E.J. (1997). Refinement of macromolecular structures by the maximum-likelihood method. Acta Crystallogr D Biol Crystallogr 53, 240-255.

Nurmohamed, S., McKay, A.R., Robinson, C.V., and Luisi, B.F. (2010). Molecular recognition between Escherichia coli enolase and ribonuclease E. Acta Crystallogr D Biol Crystallogr 66, 1036-1040.

Otwinowski, Z., Minor, W., and Charles W. Carter, Jr. (1997). Processing of X-ray diffraction data collected in oscillation mode. Methods in enzymology (Academic Press), pp. 307-326.

Pancholi, V. (2001). Multifunctional alpha-enolase: its role in diseases.
Cell Mol Life Sci 58, 902-920.

Schurig, H., Rutkat, K., Rachel, R., and Jaenicke, R. (1995). Octameric enolase from the hyperthermophilic bacterium Thermotoga maritima: purification, characterization, and image processing. Protein Sci 4, 228-236.

Seweryn, E., Pietkiewicz, J., Szamborska, A., and Gamian, A. (2007). [Enolase on the surface of prockaryotic and eukaryotic cells is a receptor for human plasminogen]. Postepy Hig Med Dosw (Online) 61, 672-682.

Smith, H.E., Damman, M., van der Velde, J., Wagenaar, F., Wisselink, H.J., Stockhofe-Zurwieden, N., and Smits, M.A. (1999). Identification and characterization of the cps locus of Streptococcus suis serotype 2: the capsule protects against phagocytosis and is an important virulence factor. Infect Immun $67,1750-1756$.

Stec, B., and Lebioda, L. (1990). Refined structure of yeast apo-enolase at 2.25 A resolution. J Mol Biol 211, 235-248.

Tang, J., Wang, C., Feng, Y., Yang, W., Song, H., Chen, Z., Yu, H., Pan, X., Zhou, X., Wang, H., et al. (2006). Streptococcal toxic shock syndrome caused by Streptococcus suis serotype 2. PLoS Med 3, e151.

Vagin, A., and Teplyakov, A. (1997). MOLREP: an automated program for molecular replacement. J Appl Crystallogr 30, 1022-1025.

Vanier, G., Sekizaki, T., Dominguez-Punaro, M.C., Esgleas, M., Osaki, M., Takamatsu, D., Segura, M., and Gottschalk, M. (2008). Disruption of srtA gene in Streptococcus suis results in decreased interactions with endothelial cells and extracellular matrix proteins. Vet Microbiol 127, 417-424.

Wang, C., Li, M., Feng, Y., Zheng, F., Dong, Y., Pan, X., Cheng, G., Dong, R., Hu, D., Feng, X., et al. (2009). The involvement of sortase A in high virulence of STSS-causing Streptococcus suis serotype 2. Arch Microbiol 191, 23-33.

Wierenga, R.K. (2001). The TIM-barrel fold: a versatile framework for efficient enzymes. Febs Lett 492, 193-198.

Xu, L., Huang, B., Du, H., Zhang, X.C., Xu, J., Li, X., and Rao, Z. (2010). Crystal structure of cytotoxin protein suilysin from Streptococcus suis. Protein Cell 1, 96-105.

Zhang, A., Chen, B., Mu, X., Li, R., Zheng, P., Zhao, Y., Chen, H., and Jin, M. (2009). Identification and characterization of a novel protective antigen, Enolase of Streptococcus suis serotype 2. Vaccine 27, 1348-1353.

Zheng, B., Tan, S., Gao, J., Han, H., Liu, J., Lu, G., Liu, D., Yi, Y., Zhu, B., and Gao, G.F. (2011). An unexpected similarity between antibiotic-resistant NDM-1 and beta-lactamase II from Erythrobacter litoralis. Protein Cell 2, 250-258. 